\title{
Patient-centred management in idiopathic pulmonary fibrosis: similar themes in three communication models
}

\author{
Wim A. Wuyts ${ }^{1}$, Fedro A. Peccatori² and Anne-Marie Russell ${ }^{3}$
}

\begin{abstract}
Affiliations: 'Unit for Interstitial Lung Diseases, Dept of Pulmonary Medicine, University Hospitals Leuven, Leuven, Belgium. ' $F e r t i l i t y$ and Procreation Unit, Division of Gynaecologic Oncology, European Institute of Oncology, Milan, Italy. ${ }^{3}$ National Heart and Lung Institute, Royal Brompton Hospital and Imperial College, London, UK.
\end{abstract}

Correspondence: Wim A. Wuyts, Dept of Pulmonary Medicine, University Hospital Leuven, Herestraat 49, 3000 Leuven, Belgium. E-mail: wim.wuyts@uzleuven.be

ABSTRACT The progressive and highly variable course of idiopathic pulmonary fibrosis (IPF) can present patients and their families with various challenges at different points of the disease. Structured communication between the healthcare professional and the patient is vital to ensure the best possible support and treatment for the patient. While research in this area has been limited, an increasing number of studies are emerging that support the role of communication in patients with debilitating and fatal lung diseases.

Communication models used in other conditions that share many challenges with IPF, such as cancer, provide important insights for developing specifically designed patient support and communications models in IPF. Three communication models will be described: 1) the patient-centred care model (for oncology); 2) the three pillars of care model (for IPF); and 3) the Brompton model of care (for interstitial lung disease). Themes common to all three models include comprehensive patient education, encouraged patient participation and an accessible healthcare system, all supported by a collaborative provider-patient relationship. The development of effective communication skills is an on-going process and it is recommended to examine communication models used in other chronic diseases.

0 @ERSpublications

Communication models in other conditions provide important insights for support and communications models in IPF http://ow.ly/uFzUS

\section{Introduction}

Idiopathic pulmonary fibrosis (IPF) is a chronic lung disease of unknown cause in which fibrotic transformation of the lung parenchyma leads to a progressive decline in lung function $[1,2]$. IPF has an extremely poor prognosis with a median survival ranging from 2.5 years to 3.5 years [3].

The relentless and often unpredictable course of IPF can present patients and their families with various challenges, which often lead to major uncertainty and fear. Studies investigating the psychological impact of living with chronic lung diseases suggest that the coping of patients with respiratory disease may, in some cases, be worse than patients with cancer [4].

Received: Feb 282014 | Accepted after revision: March 112014

Support statement: W.A. Wuyts is a Senior Clinical Investigator of the Research Foundation - Flanders (1.8.325.12N).

Conflict of interest: Disclosures can be found alongside the online version of this article at err.ersjournals.com

Provenance: Publication of this peer-reviewed article was sponsored by InterMune International AG, Muttenz, Switzerland (article sponsor, European Respiratory Review issue 132).

Copyright OERS 2014. ERR articles are open access and distributed under the terms of the Creative Commons Attribution Non-Commercial Licence 4.0. 
Effective communication between patients and caregivers is a key component in patient care but it is not always carried out satisfactorily; several patient surveys have indicated patients would prefer better communication with their doctors $[5,6]$. Structured communication between the healthcare professional and the patient is important in identifying the patients' experience/perception of their disease and in enhancing the patients' adherence to treatment, mental health and satisfaction [5-11].

Patients recently diagnosed with IPF face a range of problems, which require adequate management from the interstitial lung disease (ILD) team. Problems can be classified into several types: symptom related, treatment decisions and general information/education [12, 13]. Unfortunately, current literature covering these problems is extremely sparse.

Recently, there has been more interest in evaluating the quality of life of patients with chronic lung diseases. A recent meta-analysis of 34 papers and 3635 patients evaluated the evidence for the use of interventions in improving symptoms and quality of life in patients with fibrotic lung disease [14]. In this meta-analysis, six studies examined the effect of pulmonary rehabilitation in 194 patients with ILD. Two of the studies were randomised controlled trials (RCTs), while four were quasi-experimental open-label studies (two of which had controls). An overall improvement of $27.4 \mathrm{~m}$ was recorded in the 6-min walking distance test for patients who received pulmonary rehabilitation. The two RCTs also found an improvement in quality of life as evaluated by the Chronic Respiratory Disease Questionnaire and St George's Respiratory Questionnaire scores. The improvement in the St George's Respiratory Questionnaire score was significant ( $p=0.05)$ [14-16].

Another important element in supporting patients and an extensive and powerful tool in improving quality of life is communication. Communicating effectively is a learnt skill [6], and it is helpful to look at models of communication that have been successfully used in other diseases with similar courses and clinical outcomes to IPF. Certain cancers, such as lung cancer, share many parallels with IPF $[17,18]$ and patients face many similar challenges including poor prognoses, invasive tests and unpredictable disease courses. Both conditions require the management of patient expectations regarding treatment, emphasising delay of disease progression rather than cure. As such, a patient-centred care model that is used in oncology may have aspects which are useful in IPF [19].

Effective communication is probably one of a number of key factors by which to enhance the interaction between health professionals and patients with IPF. In order to improve the often poor quality of life in patients with this relentless disease it is important that all of these aspects are recognised [20]. Therefore, integrated models are required to facilitate the provision of best supportive care. The patient-centred care model is described below and, in addition, two other recently developed care models, the three pillars of care model for IPF patients [21] and the Brompton model of care for respiratory patients, will be discussed.

\section{Problems faced by IPF patients during the course of their disease}

Effective communication with IPF patients can be greatly improved when the healthcare provider has a thorough understanding of the problems faced by patients during the course of their disease. One way of categorising these is symptom/quality-of-life based, problems arising from the diagnostic process or those related to treatment. It should be noted that some problems may be more subtle than others. For example, one symptom/quality-of-life-based problem that many patients face is the requirement for supplemental oxygen. As might be expected, this perceived loss of independence provides logistical challenges in terms of planning excursions from home but, perhaps less obviously, the patient may also suffer psychologically as their disease becomes more visible [22]. The loss of independence may also impact on the patients' relationships as they may perceive themselves to be a burden to their family and society. There may also be financial implications if the patient is no longer able to work [12, 13, 22].

A major challenge faced by patients at the diagnostic stage is to balance the initial relief that they "do not have cancer" with the latter realisation that the prognosis is, in fact, not significantly different. In addition, the complexity of diagnosis is likely to place additional stress on the patient. A possible cause of additional stress is that $40 \%$ of patients have consulted three, or more, physicians and may have received conflicting information at various stages. An overwhelming lack of psychological support is reported by patients as they struggle to come to terms with their diagnosis and comprehend the disease process [23]. The rapidly progressive nature of IPF may sometimes present the patient with an additional challenging decision: whether or not to opt for lung transplantation $[24,25]$. Such a situation may lead to a considerable increase in anxiety. Another problem encountered by IPF patients at the treatment stage is the apparent lack of educational resources. In a recent survey of 1448 IPF patients, respondents reported a clear lack of information and resources on pulmonary fibrosis at the time of diagnosis. Information regarding treatment options, the role of supplemental oxygen, pulmonary rehabilitation and transplantation was also limited $[12,13]$. 


\section{Communication in oncology \\ Patient-centred care model}

The US National Cancer Institute (Bethesda, MD, USA) recently released a document outlining the patient care model, which focuses on patient-centred communication in cancer care [19]. The model is based on several core principles: 1) the patients' disease perspective; 2) encouragement of patients to participate in their care; 3) the creation of an optimal patient-doctor relationship; and 4) an accessible healthcare system (fig. 1). Ultimately, a strong therapeutic alliance is expected to help the physician make the best decisions on behalf of the patient and thus lead to a number of superior health outcomes. Outcomes that may be improved include the patient's management of uncertainty, their overall emotional response and information exchange between the physician and the patient (fig. 2). The patient-centred care model stresses that communication is a learnt skill and specifies various areas clinicians can focus on to help build a strong patient-doctor relationship. The use of both verbal and nonverbal behaviour by the clinician is essential in gaining the trust and confidence of the patient (table 1). There are also communication cues that may be displayed by the patient which may indicate that the patient is satisfied with the patient-doctor relationship (table 2). Guidance from the National Cancer Institute also adds that effective communication is not only reliant on the clinicians' and patients' verbal and nonverbal skills, but also on their abilities to adapt their behaviour and perspectives during the changing course of the disease.

The three pillars of care model

The three pillars of care model seeks to comprehensively identify and address the broad range of challenges faced by an IPF patient throughout the course of their disease [21]. The model defines the three pillars to be addressed as: 1) disease-centred management; 2) symptom-centred management; and 3) education and selfmanagement. Effective implementation of all three pillars is underpinned by a collaborative providerpatient partnership, which fosters the active participation of the patient in the management of their disease. The education and self-management pillar is essential in providing the patient with the knowledge required to fully participate in the decision making and management of their disease. This pillar, in particular, bolsters the provider-patient partnership as communication between both parties helps the patient to gain perspective on their disease and thereby set realistic goals, make appropriate decisions, stay in control of their care and prepare for the future. It is recommended that patient education be started immediately upon diagnosis and that the information is tailored according to the needs of the patient. Topics that should be covered include disease pathology, course and prognosis. Disease-centred management comprises pharmacological and non-pharmacological treatment, whereas symptom-centred management focusses on dyspnoea, cough, deconditioning and anxiety. The patient should be informed in a timely manner about the possible treatment options for the disease.

Advanced care planning involves setting treatment goals that are consistent with the patients' values and preferences. Advanced care planning is an integral part of the education and self-management pillar due to the progressive nature of IPF. End-of-life care planning in IPF should occur at a non-critical time (when death is not impending) and should take into account the patient's views and wishes as much as possible.

The three pillars of care model acknowledges that the nature of the problems presented to an IPF patient is likely to change with time. For example, in the early stage of IPF, a disease-centred approach whereby the patient and physician consider decisions on IPF treatments is likely to be relevant, while later in the course of the disease decision making it is likely to be more focused on palliative therapies. It is crucial during this process that both approaches are initiated together at the time of diagnosis. Patient education should attempt to cover all aspects of this spectrum. To reflect the dynamic nature of problems, the model proposes that active discussion of goals should occur at regular intervals to continually allow for reassessment of the patients' needs and treatment goals during the disease course.

\section{Brompton model of care}

Patient satisfaction with medical care and disease education is reported to be higher in patients who receive care at a recognised centre of excellence [13]. NHS England's service specification acknowledges the importance of multidisciplinary team (MDT) input to assign the correct diagnoses and initiate the most appropriate therapy for patients with ILD [26]. In the 1960s, the Royal Brompton Hospital (London, UK) was ahead of its time, bringing together clinical- and laboratory-based researchers to work together as teams. Integrated thinking enabled the set-up of a joint management clinic with the Royal Marsden Hospital (London, UK) for patients with lung cancer. Margaret Turner-Warwick, the first full-time female chest physician for adults at the Royal Brompton Hospital, was committed to collaborative working and adapted the Brompton-Marsden model of care that underpins the model used today in the interstitial lung disease unit at the Royal Brompton Hospital [27]. Embedded in this is a holistic approach enabling individualised management of the patient. 


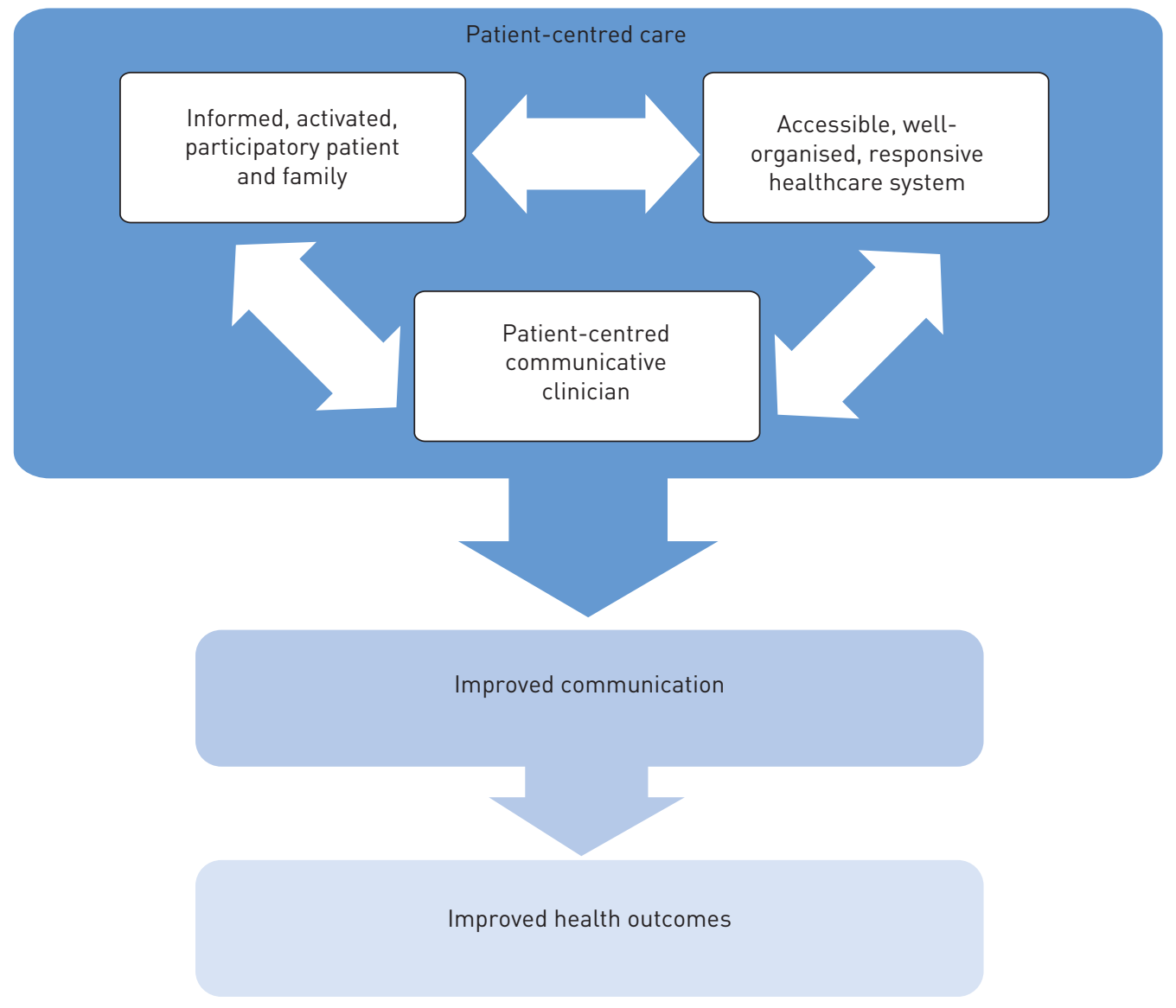

FIGURE 1 Patient-centred care model in oncology. Reproduced from [19] with permission from the publisher.

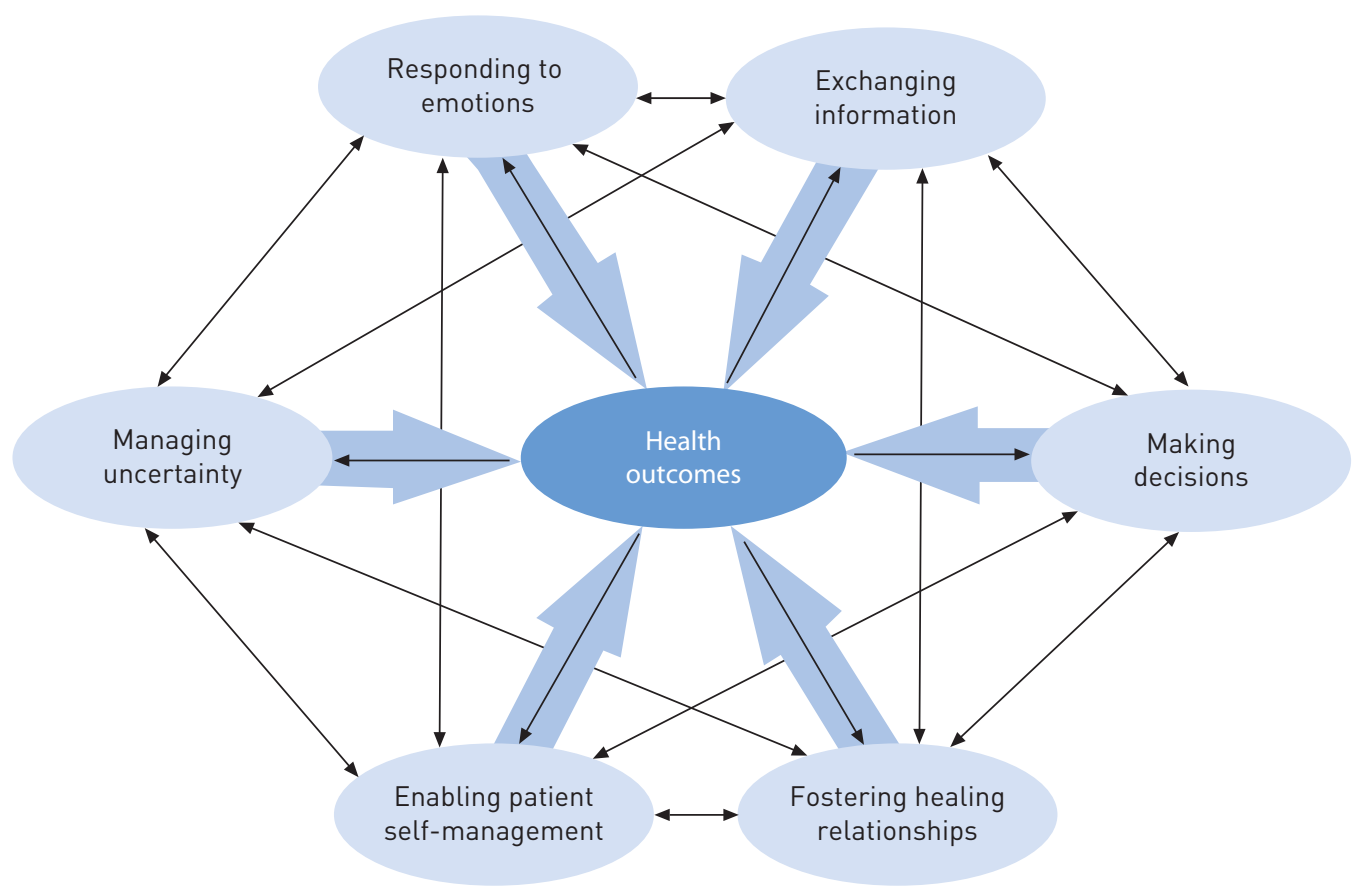

FIGURE 2 Patient-centred care model in oncology: potential health outcomes from better communication. Reproduced from [19] with permission from the publisher. 


\section{TABLE 1 Patient-centred clinician behaviours}

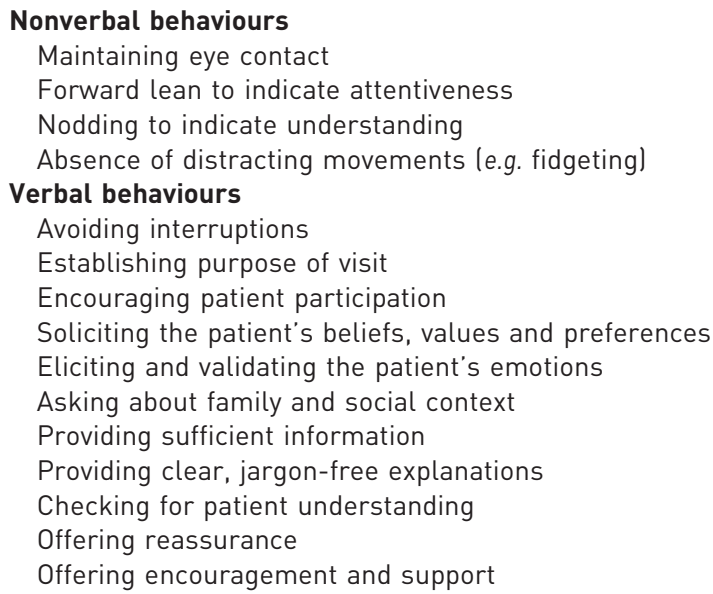

Reproduced from [19] with permission from the publisher.

There were approximately 550 referrals to the interstitial lung disease unit in 2011 increasing to 590 cases in 2012 (fig. 3). The Brompton model of care provides diagnostic work-up and multidisciplinary treatment of chronic respiratory diseases within a single tertiary-care centre. This unified provision of services accelerates the diagnostic process and delivers a timely, accurate diagnosis to the patient thereby reducing anxiety.

Patients are seen within 6 weeks of referral and, in cases where probable, possible or definite IPF is suspected, patients are admitted to the ward for $48-72 \mathrm{~h}$ for a comprehensive diagnostic work-up. This relieves the burden of multiple journeys for these patients whose independence may be compromised by symptoms such as breathlessness and chronic fatigue.

Following this diagnostic work-up patients and their relatives are invited to attend a ward round discussion with members of the interdisciplinary team. As the model aims to provide all care services for the patient at one centre, the interdisciplinary team is comprehensive and includes healthcare professionals who are not routinely involved in the care of IPF patients in many clinics. Palliative care specialists, dieticians, rheumatologists and clinical nurse specialists contribute to these meetings. Input from a psychologist is available on a case-by-case basis. Where diagnostic uncertainty exists, additional comprehensive discussion takes place with core members of the MDT (fig. 4). The integration of members of the interdisciplinary team into the formal MDT meetings is under review as is the inclusion of a patient advisor.

Patients are encouraged to participate in research. "ILD patient participation and involvement" forums are held periodically in our Education Unit within the clinical research facility at the Royal Brompton Hospital, and the clinical nurse specialist has recently set up a monthly ILD support group. Attending patients can listen to speakers who discuss topics relevant to ILD research and treatment. Patients report that an awareness of research assists in the decision making processes of their care.

\section{TABLE 2 Active patient communication behaviours}

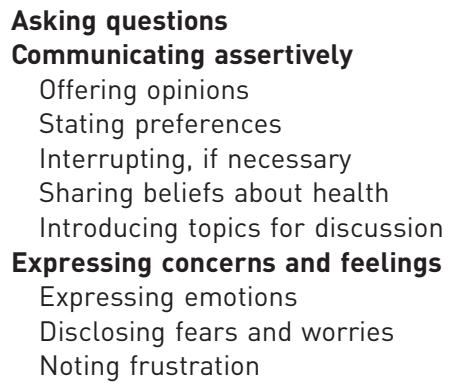

Reproduced from [19] with permission from the publisher. 


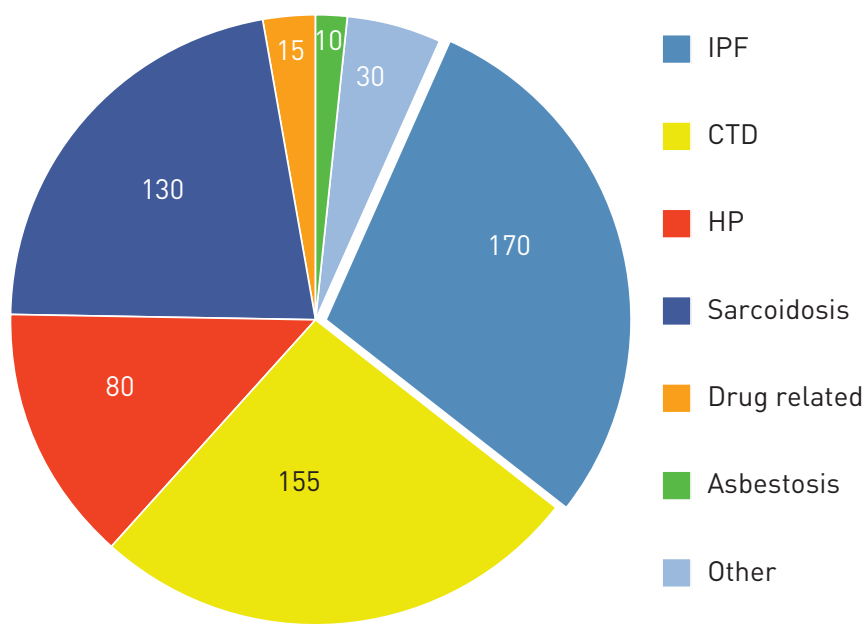

FIGURE 3 The Brompton model of care. Referrals to the interstitial lung disease unit at the Royal Brompton Hospital (London, UK) in 2012. IPF: idiopathic pulmonary fibrosis; CTD: connective tissue disease; HP: hypersensitivity pneumonitis.

Attendance at the interstitial lung disease unit is likely to be reassuring for the patient and their families. Hospital Anxiety and Depression Scale scores collected in a cohort of 150 IPF patients at the Royal Brompton Hospital over 12 months found anxiety scores were reduced in this time period. However, depression scores were increased, independently of disease severity [28].

The Brompton model of care offers patients access to highly trained professionals who understand their disease and its process. Patients receive individualised care and information according to the stage of disease progression. The importance of good access to a knowledgeable healthcare professional is valued by the patients and their families and may account for the observed reduction in patient anxiety scores. Some members of staff offer unrestricted access to additional support via email.

Core MDT
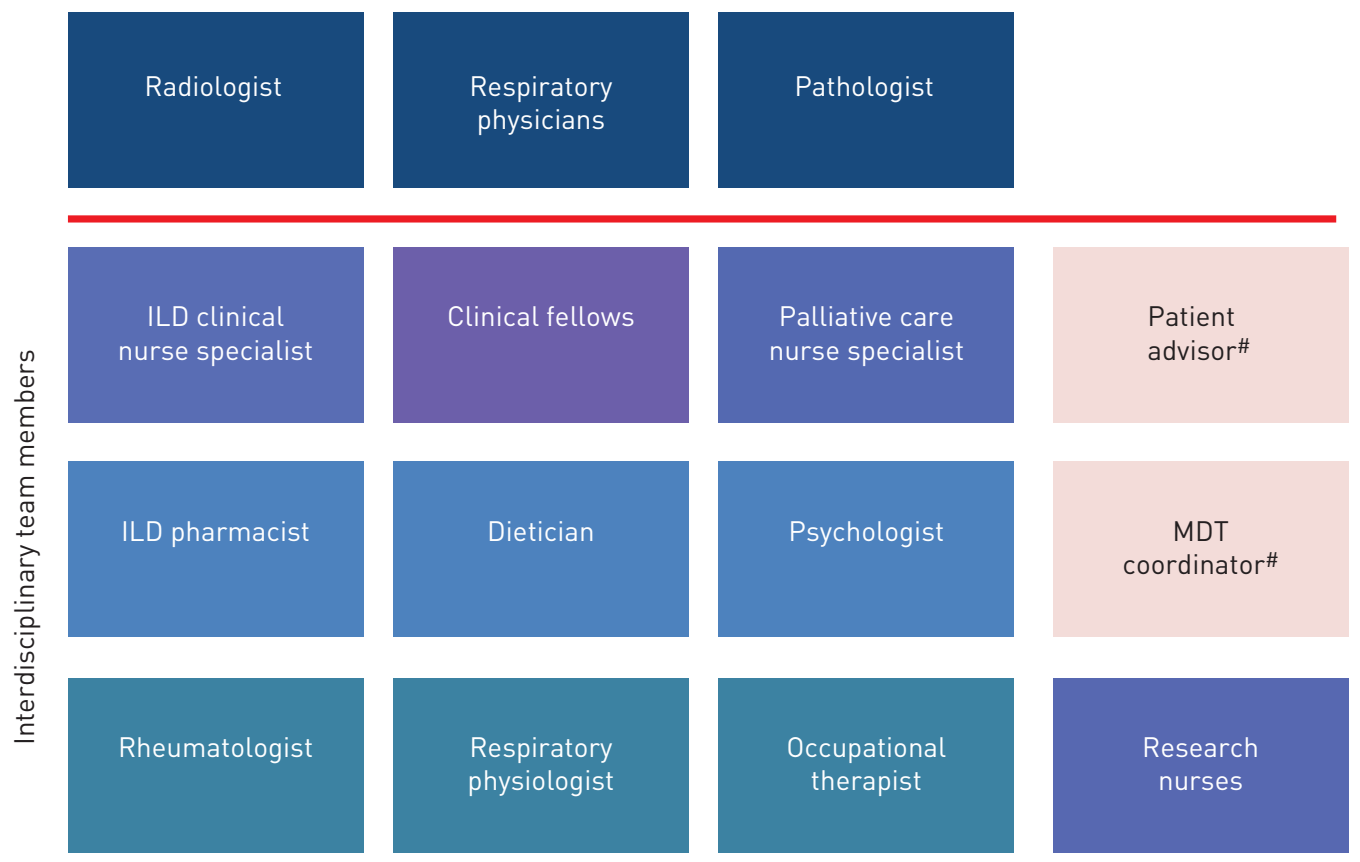

FIGURE 4 The Brompton model of care: core members and interdisciplinary team members for interstitial lung disease (ILD) patients. MDT: multidisciplinary team. ${ }^{*}$ : to be appointed. 


\section{Conclusion}

The unpredictable and frequently rapidly progressive nature of IPF means that patients and their families are often faced with a number of challenges at different points of the disease course. Therefore, efficient communication between the healthcare professional and the patient is of paramount importance in helping the patient and their family cope and feel supported in the disease process.

Communication in such situations involves specific skills that can be learnt and should be tailored to the individuals' needs. There is recent evidence to suggest that interventions such as starting up support groups may have a positive impact on the outcomes in patients with fibrotic interstitial lung disease.

There may be shared lessons from communication models used in other diseases similar to IPF, which may help improve communication and patient outcomes in IPF. Themes common to the three models of care studied in this paper (the patient-centred care model, the three pillars of care model and the Brompton model of care) include strong patient education, encouraged patient participation and an accessible healthcare team, underpinned by a strong provider-patient relationship. Ward rounds offering the patient individualised support and annual research and treatment forums (as in the Brompton model of care) are initiatives for simultaneous patient participation and patient education. The "accessible, well organised and responsive healthcare system" described in the patient-centred oncology model is exemplified by the Brompton model of care (fig. 4), which provides a rapid and complete diagnostic work-up under one roof, a structured ward round and a comprehensive MDT, some of whom are accessible $24 \mathrm{~h}$ a day.

The treatment strategy for IPF has changed over time from treatments directed towards the disease to palliative therapy. Advanced care planning should occur early in the disease process in order to best comply with the patients' values and wishes. Regular meetings between the healthcare professional and the patient throughout the disease course help both parties react to the changing nature of problems and further strengthens the provider-patient relationship.

A patient-centred communication approach ultimately aims to make the patient feel as comfortable and listened to as possible so they can best express their wishes and feel confident to participate in the decisionmaking process. The advantages and disadvantages of integrating the patient into the MDT team is currently under consideration for the Brompton care model. The learning and practice of communication skills is an on-going process. In future, it may be helpful to look at communication models used in other chronic diseases.

\section{Acknowledgements}

This article is based on the proceedings of the 2013 Advancing IPF Research (AIR) meeting (Nice, France), which was sponsored by InterMune International AG (Muttenz, Switzerland). Medical writing support was provided by Michael Smith (IntraMed International, Milan, Italy), which was funded by InterMune International AG.

\section{References}

Zolak JS, de Andrade JA. Idiopathic pulmonary fibrosis. Immunol Allergy Clin North Am 2012; 32: 473-485.

2 Raghu G, Collard HR, Egan JJ, et al. An Official ATS/ERS/JRS/ALAT statement: idiopathic pulmonary fibrosis: evidence-based guidelines for diagnosis and management. Am J Respir Crit Care Med 2011; 183: 788-824.

3 Ley B, Collard HR, King TE Jr. Clinical course and prediction of survival in idiopathic pulmonary fibrosis. Am J Respir Crit Care Med 2011; 183: 431-440.

4 Gore JM, Brophy CJ, Greenstone MA. How well do we care for patients with end stage chronic obstructive pulmonary disease (COPD)? A comparison of palliative care and quality of life in COPD and lung cancer. Thorax 2000; 55: 1000-1006.

5 Farin E. Die Patient-Behandler-Kommunikation bei chronischen Krankheiten: Uberblick uber den Forschungsstand in ausgewahlten Themenbereichen [Patient-provider communication in chronic illness: current state of research in selected areas]. Rehabilitation (Stuttg) 2010; 49: 277-291.

6 Ha JF, Longnecker N. Doctor-patient communication: a review. Ochsner J 2010; 10: 38-43.

7 Brédart A, Bouleuc C, Dolbeault S. Doctor-patient communication and satisfaction with care in oncology. Curr Opin Oncol 2005; 17: 351-354.

8 Arora N. Interacting with cancer patients: the significance of physicians' communication behavior. Soc Sci Med 2003; 57: 791-806.

9 Ong LM, de Haes JC, Hoos AM, et al. Doctor-patients communication: a review of the literature. Soc Sci Med 1995; 40: 903-918.

10 Platt FW, Keating KN. Differences in physician and patient perceptions of uncomplicated UTI symptom severity: understanding the communication gap. Int J Clin Prac 2007; 61: 303-308.

11 Harmon G, Lefante J, Krousel-Wood M. Overcoming barriers: the role of providers in improving patient adherence to antihypertensive medications. Curr Opin Cardiol 2006; 21: 310-315.

12 Collard HR, Tino G, Noble PW, et al. Patient experiences with pulmonary fibrosis. Respir Med 2007; 101: 1350-1354.

13 Schoenheit G, Becattelli I, Cohen AH. Living with idiopathic pulmonary fibrosis: an in-depth qualitative survey of European patients. Chron Respir Dis 2011; 8: 1-7.

14 Bajwah S, Ross JR, Peacock JL, et al. Interventions to improve symptoms and quality of life of patients with fibrotic interstitial lung disease: a systematic review of the literature. Thorax 2013; 68: 867-879. 
Nishiyama O, Kondoh Y, Kimura T, et al. Effects of pulmonary rehabilitation in patients with idiopathic pulmonary fibrosis. Respirology 2008; 13: 394-399.

16 Holland AE, Hill CJ, Conron M, et al. Short term improvement in exercise capacity and symptoms following exercise training in interstitial lung disease. Thorax 2008; 63: 549-554.

17 Vancheri C. Common pathways in idiopathic pulmonary fibrosis and cancer. Eur Respir Rev 2013; 22: $265-272$.

18 Vancheri C, Failla M, Crimi N, et al. Idiopathic pulmonary fibrosis: a disease with similarities and links to cancer biology. Eur Respir J 2010; 35: 496-504.

19 Epstein RM, Street RL Jr. Patient-Centered Communication in Cancer Care: Promoting Healing and Reducing Suffering. National Cancer Institute, NIH Publication No. 07-6225. Bethesda, MD, 2007.

20 Lindell KO, Olshansky E, Song MK, et al. Impact of a disease-management program on symptom burden and health-related quality of life in patients with idiopathic pulmonary fibrosis and their care partners. Heart Lung 2010; 39: 304-313.

21 Lee JS, McLaughlin S, Collard HR. Comprehensive care of the patient with idiopathic pulmonary fibrosis. Curr Opin Pulm Med 2011; 17: 348-354.

22 Giot C, Maronati M, Becattelli I, et al. Idiopathic pulmonary fibrosis: an EU patient perspective survey. Curr Respir Med Rev 2013; 9: 112-119.

23 Russell AM, Vancheri C, Maronati M, et al. A qualitative European survey of patients perceptions of current management of idiopathic pulmonary fibrosis. Thorax 2013; 68: A166-A167.

24 De Vries J, Kessels BL, Drent M. Quality of life of idiopathic pulmonary fibrosis patients. Eur Respir J 2001; 17: 954-961.

25 Vermeulen KM, Bosma OH, Bij Wv, et al. Stress, psychological distress, and coping in patients on the waiting list for lung transplantation: an exploratory study. Transpl Int 2005; 18: 954-959.

26 NHS England. A14/S/c. 2013/14 NHS Standard Contract For Respiratory: Interstitial Lung Disease (Adult). www. england.nhs.uk/wp-content/uploads/2013/06/a14-respiratory-inters-lung.pdf Date last updated: October 1, 2013. Date last accessed: December 12, 2014.

27 Turner-Warwick M. Living Medicine: Recollections and Reflections. London, Royal college of Physicians, 2005.

28 Russell AM, Fraser U, Molyneaux P, et al. Quality of life measures in patients with idiopathic pulmonary fibrosis. Eur Respir J 2013; 42: Suppl. 57, 688s. 